\title{
COMPARATIVE ANALYSIS OF ACOUSTIC EMISSION IN PLAIN AND REINFORCED CONCRETE USING WAVELET TRANSFORM
}

\author{
NITIN B. BURUD*, J. M. CHANDRA KISHEN ${ }^{\dagger}$ \\ *Indian Institute of Science \\ Bangalore, India \\ e-mail: nitinburud@gmail.com \\ $\dagger$ e-mail: chandrak@civil.iisc.ernet.in
}

Key words: Reinforced Concrete, Acoustic Emission, Time-Frequency Analysis, Wavelet Transform

\begin{abstract}
The present work is a comparative study of Acoustic Emission (AE) signals observed during three point bending tests of plain and reinforced concrete beam specimens. AE signals were recorded throughout the tests and analyzed using Wavelet Packet Decomposition (WPD). One of the objective of current study is to utilize available spectral information of signals for comparative study of AE from reinforced concrete and plain concrete. A classification method for AE signals based on sub-band energy ratios obtained from WPD is proposed. Also, use of relative sub-band energy distribution as a Acoustic emission signature for concrete is proposed. Differences in AE signals of reinforced and plain concrete are highlighted at sub-band level. It is concluded that yielding of reinforcing steel has contributed in higher sub-bands $78.126-250 \mathrm{kHz}$ of AE signals.
\end{abstract}

\section{INTRODUCTION}

Progressive structural damage is always an issue of concern for engineers. Though structures are designed for desired service loads, there is always a possibility of early failure due to progressive damage. Causes of damage can be fatigue, creep, shrinkage, settlement, environmental effects, earthquakes etc. Damage grows transiently causing loss of strength in structures leading it to ultimate failure. Usually cracks and large deformations are visual indications of growing damages. Apparently these damage indicators become visible, by the time an irreparable damage might be prevalent in the structure. Hence, monitoring of growing cracks as one of the damage indicator can be effectively considered for structural health assessment.

In the cognizance of structural health monitoring, Acoustic Emission (AE) monitoring has seen appreciable amount of development in last few decades [1,2]. Acoustic emissions are elastic waves produced by cracking of material and are captured by piezoelectric sensors mounted on the surface. Rate of incoming signals, as well as characteristics like their amplitude, energy and frequency content are well correlated to the density and severity of cracks. For the well behaved materials like metals and ceramics, AE monitoring has been proved efficient and accurate for source localization and characterization problems. But due to inherent inhomogeneity in cementitious materials like concrete, such accuracy has not been yet achieved. Crack initiation and propagation in concrete is caused by many complex phenomenons such as cement matrix cracking, aggregate cracking, debonding of aggregates, debonding of reinforcement, friction between fractured and interlocked surfaces etc. Inherent heterogeneous and anisotropic nature of concrete makes acoustic emission waves attenuate and scatter. 
Currently, parameters from stress wave signals in time domain can be extracted and related to damage of the structure. Time domain parameters vary with the signal strength and are affected by noise. For example, as rate of loading increases, the possibility of acquiring high strength signals is more due to increased rate of cracking. Also, the duration of signal can appear to increase due to acquisition of overlapped signals and their reflections. Consequently, conventional AE time domain parameters are unreliable to classify signals and its sources. Many methods have been proposed in the literature which are based on conventional time domain parameters for classification of signals [3, 4].

On the other hand, signal based approach requires more computational efforts and time. Spectral information can be more useful for quantitative analysis of AE signal. Spectral characteristics will not change much due to increased rate of loading and multiple reflection as the cracking material is same and eventually the AE signature will be the same. Higher frequencies may attenuate faster as the distance between source and sensor increases. But higher frequencies are minor components of larger amplitude signals in concrete. Hence, a method can be devised based on the relative sub-band energy distribution of signals. Such unique relative distribution of energy can be used as AE signature. A library based approach should be adopted to correlate relative energy distribution to the behavior of concrete based on AE signature. To compute relative distribution of sub-band energy in spectral domain, a recently developed Wavelet packet decomposition (WPD) [5] has been used in this work. Many researchers have applied wavelet transform to acoustic emission for signal detection, denoising and classification [6-10] successfully.

Utilization of available spectral information for comparative understanding of $\mathrm{AE}$ signals in reinforced and plain concrete is one of the objective of this study. AE signals are classified based on sub-band energy distribution obtained from wavelet packet decomposition. Use of relative energy distribution as $\mathrm{AE}$ signature has been proposed in the present work. Differences between reinforced and plain concrete are highlighted at sub-band level.

\section{Acoustic Emission Signature}

According to ASTM standards [11], AE signature can be defined as a characteristic set of reproducible attributes of acoustic emission signals associated with a specific test article as observed with a particular instrumentation system under specified test conditions. The correlation of acoustic emission features to specific AE pattern is termed as AE signature analysis. Acoustic emission signature is derived either from the time domain parameters or from the frequency domain features of AE signals. Boyce et al. [12] have investigated acoustic emission in rocks and provided extension of Mogi's [13] work which represented the $\mathrm{AE}$ signature as stress versus AE response curves. Clough et al. [14] proposed a method based on indentation to produce reproducible signals from defects of steel alloy and termed such signals as AE signature. AE signature concept is also adopted in applications for condition monitoring in which $\mathrm{AE}$ signal are continuous in time rather than small duration bursts. Ativitavas [15] used slope of cumulative amplitude distribution (b-value) for AE signature analysis in fibre reinforced plastic. Mirmiran and Philip [16] proposed Specific Acitivity ratio as the measure to compare various AE signature in concrete. Specific activity ratio is defined as observed total $\mathrm{AE}$ counts, total AE energy and peak amplitude normalized with respect to failure load of the beam. A wide range of $\mathrm{AE}$ signature analysis techniques has been proposed in literature.

Authors of the present work have observed highly correlated AE signals from a single specimen during fracture process of concrete. $\mathrm{AE}$ signals depend on chemical composition, particle size distribution and mode of fracture. If mode of fracture and particle size distribution is kept constant then such inter-correlated AE signals can be obtained from different specimen. 
Hence such signals can be used for classification and characterization of AE sources in material. This has motivated the authors to define a new AE signature as spectral energy distribution. In the present work, spectral energy of $\mathrm{AE}$ signals is divided in to sub-bands and then normalized with total energy. Such relative energy distribution in sub-bands can be used as AE signature. Each source in material will have different signature by which different sources can be identified separately. Such signatures can be also used to extract AE signal from noisy data.

\section{Wavelet Packet Decomposition}

Acoustic emission signals are narrow banded and non-stationary in nature. A signal is stationary if its frequency or spectral contents are unchanged with respect to time. Hence signal processing technique for $\mathrm{AE}$ analysis demands better resolution in frequency domain. Fourier transform (FT) uses sine or cosine waves as the basic functions to represent signals in frequency domain. But due to sine and cosine functions as basis, stationarity assumption prevails in FT. Also, such basis functions are not localized in time which makes FT unsuitable to localize signal frequency as a function of time. A straight forward solution for this limitation of FT is to introduce an analysis window of certain length that slides through the signal along the time axis to perform a time-localized Fourier transform. This concept is well known as Short time Fourier transform (STFT). But, selection of a suitable window size for effective signal decomposition using the STFT technique is not guaranteed. Recently developed Wavelet Transform (WT) is now widely used to analyze time series that contain non-stationary power at many different frequencies.

Wavelet Transform (WT) technique has been proved more advantageous over the Short time Fourier Transform (STFT) technique for better resolution required for time localization of frequency. WT uses time localized basis function and decomposes a signal into its components. In the case of wavelet analysis, the basic functions consist of the wavelet scale function, and scaled and shifted versions of the mother wavelet. Also, WT offers wide range of basis function according to the requirement of the signal to be analyzed. WT enables analysis of data at multiple levels of resolution. Details of wavelet transform can be referred from [5]. Many different types of wavelet transforms are available for specific purposes and Wavelet packet decomposition (WPD) is one of them.

In WPD, a signal is split into its approximate and detailed component using dyadic filter bank. The dyadic filters are nothing but lowpass and high-pass filters with complementary bandwidths also known as a quadrature mirror filter (QMF) pair. Low-pass filter produces approximation and high-pass filter produces details of the signal. The approximation is then itself split into a second level approximation and detail. The same procedure is followed on first level detail component to produce second level approximation and detail from first level details. For $j$ th level decomposition, WPD produces $2^{j}$ components of the signal. These components are referred as nodes for simplicity. A wavelet decomposition tree for three levels decomposition is shown in Figure 1. For further detailed description of wavelet decompostion [17] may be referred.

\section{Experimental Setup}

For comparative study, three beam specimens each of plain and reinforced concrete were tested. Beams of $75 \mathrm{~mm}$ depth, $50 \mathrm{~mm}$ width and $333.75 \mathrm{~mm}$ length were tested under three point bending. Reinforced concrete beams were provided with a $6 \mathrm{~mm}$ diameter bar just above the notch tip. A computer controlled servo hydraulic machine was employed for testing under crack mouth opening displacement (CMOD) control. Loading rate was set to $1 \mu \mathrm{m} / \mathrm{sec}$ for both specimen. Center point deflection of beams was measured using a linear variable differential transformer (LVDT) of $5 \mathrm{~mm}$ range, while load was recorded using load cell of $40 \mathrm{kN}$ capacity. A Physical Acoustic Corporation (PAC) system was used to monitor the Acoustic Emission (AE) throughout the 
test. Four resonant type R6D AE sensors were mounted on beams as shown in Figure 2. AE sensors with resonant frequency around $55 \mathrm{kHz}$ was used to acquire AE waveforms. Due to weak strength of $\mathrm{AE}$ signals, preamplifier with $40 \mathrm{~dB}$ gain was set for signal amplification. A threshold limit was set to $45 \mathrm{~dB}$ for background noise reduction. Sampling rate of $1 \mathrm{MHz}$ was used to ensure good time and frequency signal resolution. All waveforms crossing threshold level were stored digitally. Waveforms below threshold level were neglected.

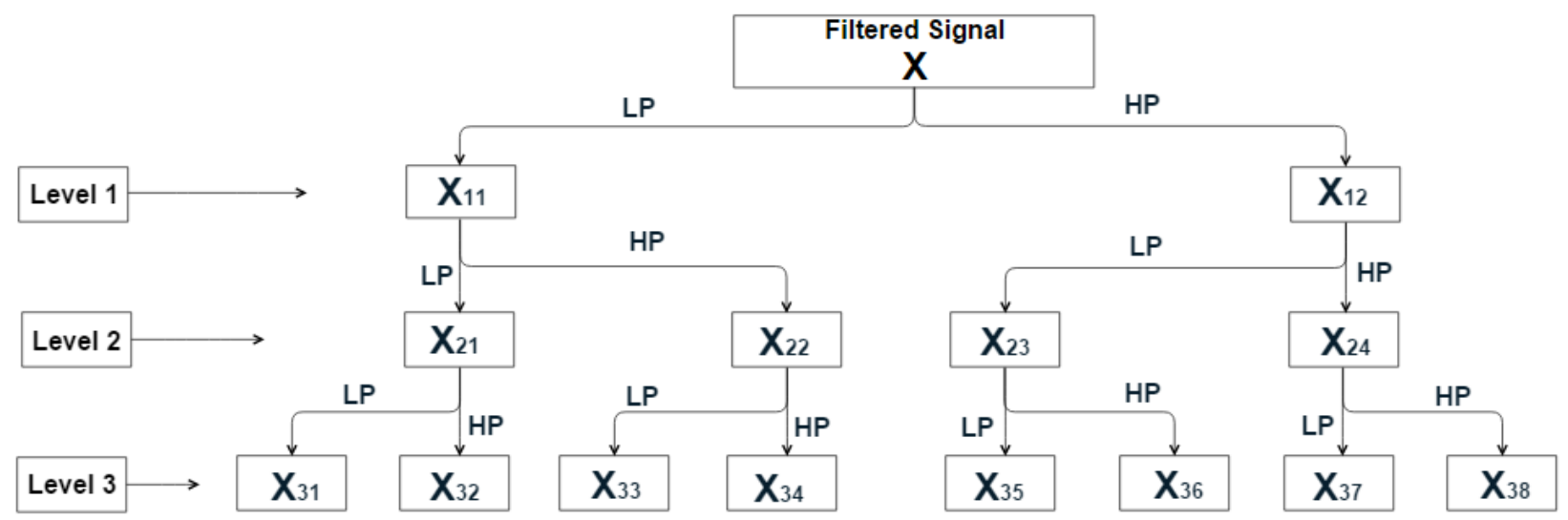

Figure 1: Wavelet Decomposition Tree.( LP-Low-pass, HP-High-pass)

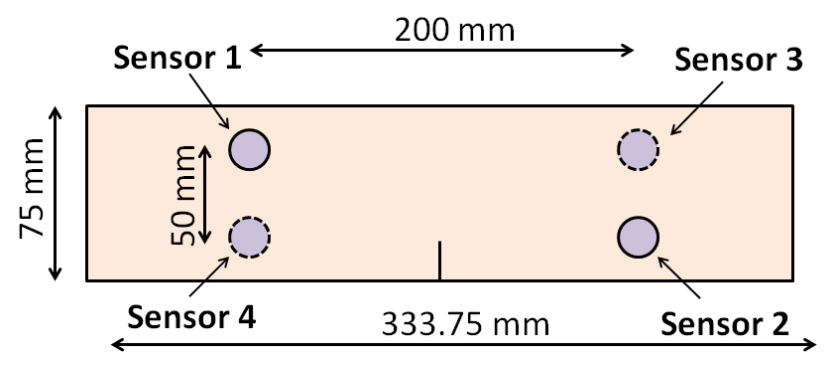

Figure 2: Beam dimensions with AE sensors.
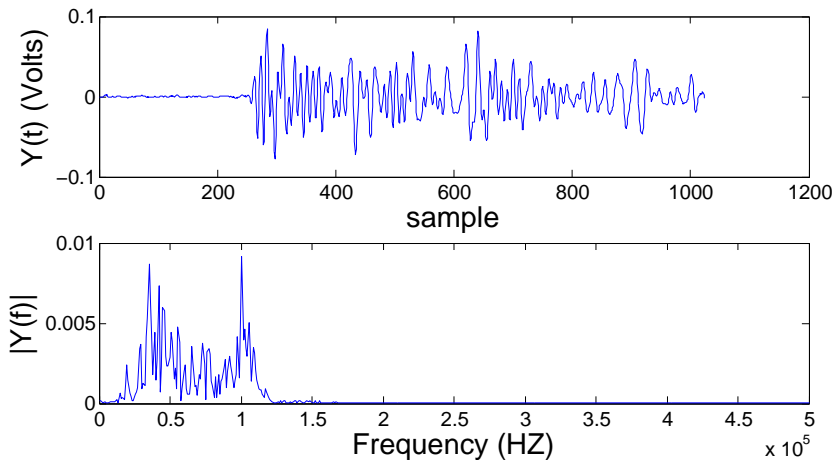

Figure 3: Typical AE signal from concrete

\section{Signal Processing}

According to Nyquist's sampling theorem, the maximum frequency which can be acquired by $1 \mathrm{MHz}$ sampling rate is about $500 \mathrm{kHz}$ (i.e. half of the sampling frequency). Though acquired AE signals are narrow banded, the sampling rate of about $500 \mathrm{kHz}$ is sufficient to acquire highest frequency in the signals. High sampling rate was used in present study for better time resolution. Due to use of resonance type sensors the frequency response is not flat in the frequency range of sensor. Hence deconvolution was applied to signals to remove unwanted effect of sensors using frequency response curve provided by the PAC along with sensor. A typical AE signal from concrete with its amplitude spectra is shown in Figure 3. A low pass filter with cutoff frequency around 250 $\mathrm{kHz}$ was used to remove unwanted noise from signals. The tests were performed in isolated laboratory which helped to avoid environmental noises and also resulted in high signal to noise ratio. Filtered signals were further decomposed in to sub-bands using 'Daubechies 
db20' wavelet. The WPT gave $2^{j}$ sub-bands at $\mathrm{j}$ th level decomposition with frequency spacing of Fs/ $(2 j+1)$, where Fs is sampling frequency.

\section{Relative Wavelet Energy}

Wavelet packet decomposition was used to discretize signals in sub-bands. If $X(t)$ is a AE signal, then it is possible to decompose it into components, where $X_{j 1}, X_{j 2}, \ldots X_{j k}$ are the components of jth level and $k$ represents the node number of the decomposed signal as shown in Figure 1. Energy of each component can be defined as $\mathrm{E}_{j 1}, \mathrm{E}_{j 2}, \ldots \ldots . \mathrm{E}_{j k}$ at jth level. Normalized node energy with respect to total energy gives relative wavelet energy. The relative wavelet energy ratio vector $\mathrm{r}_{j k}$ represents energy in decomposed sub-bands.

The total energy of the signal is:

$$
E_{\text {Total }}(t)=\sum E_{j k}(t)
$$

The ratio of energies at different levels to the total energy is considered to determine energy distribution at different components. Hence, the relative wavelet energy can be written as:

$$
r_{j k}=\frac{E_{j k}(t)}{E_{\text {Total }}(t)}, k=1, \ldots 2^{j}
$$

For the present study, 4th level decomposition was performed which resulted in 16 subband components of signal. The frequency spacing is $15.625 \mathrm{kHz}$ for each sub-band with maximum $250 \mathrm{kHz}$ frequency. These sub-bands are often referred as nodes. For detailed frequency content of these sub-bands refer table 1 .
Table 1: Frequency content of sub-bands.

\begin{tabular}{|l|c|}
\hline Frequency $\mathrm{kHz}$ & sub-band/node \\
\hline $0-15.625$ & 1 \\
\hline $15.626-31.25$ & 2 \\
\hline $31.251-46.875$ & 3 \\
\hline $46.876-62.5$ & 4 \\
\hline $62.501-78.125$ & 5 \\
\hline $78.126-93.75$ & 6 \\
\hline $93.751-109.375$ & 7 \\
\hline $109.376-125$ & 8 \\
\hline $125.001-140.625$ & 9 \\
\hline $140.626-156.25$ & 10 \\
\hline $156.251-171.875$ & 11 \\
\hline $171.876-187.5$ & 12 \\
\hline $187.501-203.125$ & 13 \\
\hline $203.126-218.75$ & 14 \\
\hline $218.751-234.375$ & 15 \\
\hline $234.376-250$ & 16 \\
\hline
\end{tabular}

\section{Results and Discussion}

CMOD control allows stable crack growth in notched concrete beam specimens. The tested plain concrete specimens showed postpeak softening behavior until the failure. While reinforced concrete beams could not reach final failure due to its crack mouth opening displacement exceeded the range of CMOD clip gauge. But considering practical situation of real structures, large crack mouth opening which exceeded $5 \mathrm{~mm}$ can be considered as failure of reinforced beams for presented experiments. Typical load deflection curve for plain and reinforced concrete is shown in Figure 4

Time duration of reinforced beams to reach failure is much greater than plain concrete beams which indicates that the crack growth rate is much slower in reinforced beams due to yielding of steel. Large amount of energy is required to deform the reinforcement steel. The average load carried by plain concrete beams is $3.30 \mathrm{kN}$ and by reinforced concrete beams is about $9.40 \mathrm{kN}$. 


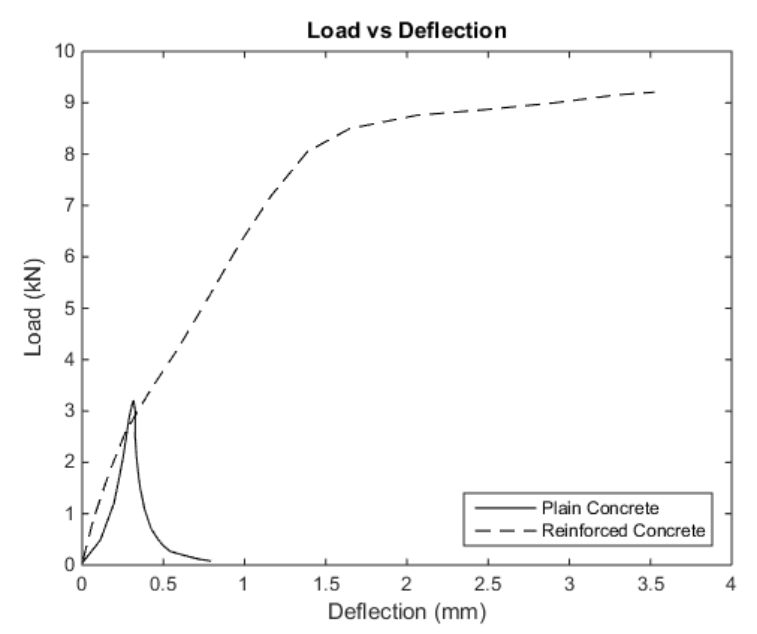

Figure 4: Final failure of plain concrete beam.

Notch is provided to ensure the propagation of single dominant crack at the center of beam specimen which is easier to monitor using acoustic emission. This also helps to relate the area of cracked surface to the released energy, as there is only a single dominant crack rather than dispersed cracks which generally appear in specimens without a notch. In this context, dispersed cracks are multiple flexure, shear and flexure-shear cracks which appear at the bottom of reinforced beams. There is a possibility of dispersed cracking in reinforced beams if the section is over reinforced but in the presented study the section is designed as under reinforced. Crack length or cracked surface area for both type of specimens is almost the same due to presence of notch at center. The cracked specimens are shown in Figure 5 and 6 .

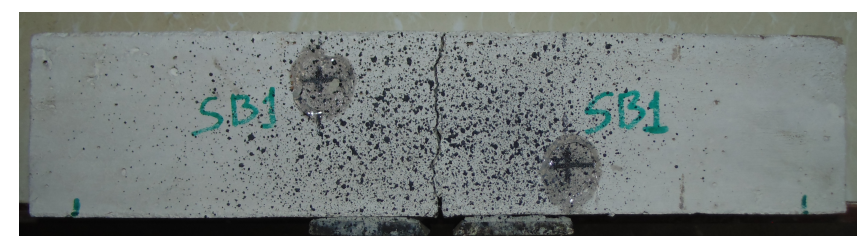

Figure 5: Final failure of plain concrete beam.

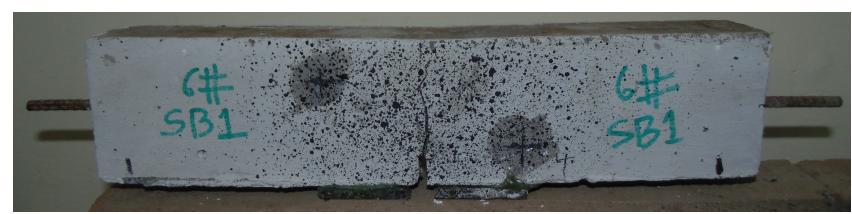

Figure 6: Final failure of reinforced concrete beam.

The energy ratios determined using wavelet packet decomposition represents distribution of energy over the sub-bands of the signal as shown in Figure 7 and 8 for plain and reinforced concrete respectively.
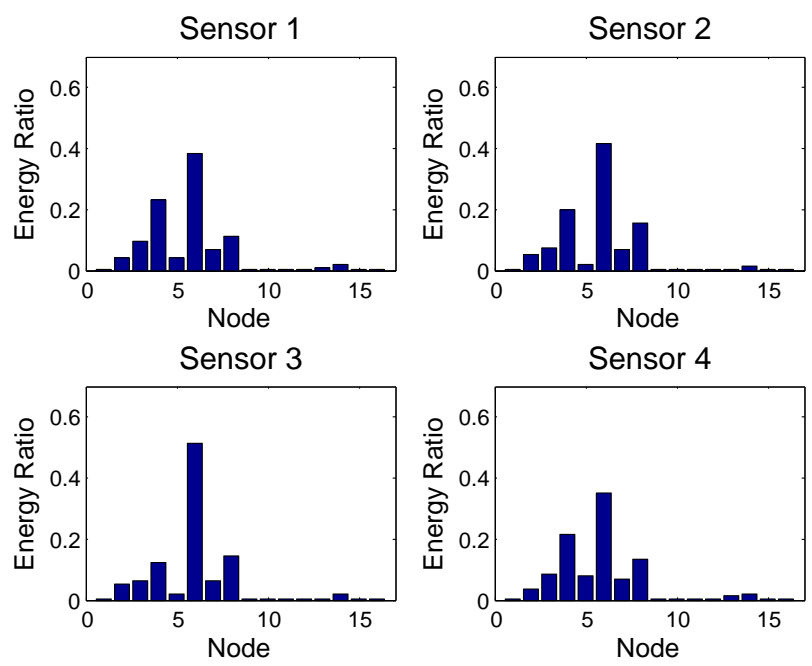

Figure 7: Energy distribution for plain concrete beams.
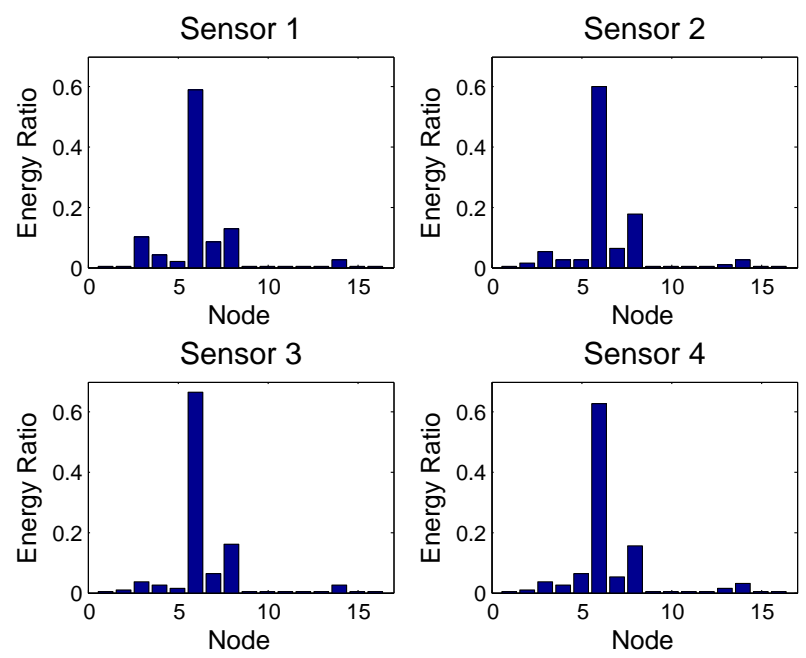

Figure 8: Energy distribution for reinforced concrete beams. 
To acquire AE signal from steel, broad band sensors or sensors designed specially for AE of steel needs to be used. AE of concrete belongs to lower frequency ranges than steel. Though steel emits $\mathrm{AE}$ up to $1 \mathrm{MHz}$, this does not mean that lower or higher frequencies will not be present. These lower frequencies from steel can be acquired by the resonant type sensors used in present work.

Energy ratios are calculated only for AE hits which are acquired by all the four sensors and can be recognized as events. A total of 9008 and 55627 events were recorded for plain and reinforced concrete specimen respectively. It is obvious that number of events in reinforce concrete will be much higher than plain concrete due to slow cracking rate in reinforced concrete. In both type of specimen, energy contribution by $6^{\text {th }}$ sub-band (i.e. $78.125-93.75 \mathrm{kHz}$ ) is dominant. This can be considered as major contribution by concrete cracking. Then $4^{\text {th }}$ sub-band in plain concrete and $8^{t h}$ sub-band in reinforced concrete dominates next to the $6^{\text {th }}$ sub-band. The maximum contribution of 6th sub-band is $40 \%$ in plain concrete and is almost $60 \%$ in reinforced concrete. The reason behind this difference can be due to additional contribution of stress waves emanating from yielding of reinforcing steel. The maximum frequency of $\mathrm{AE}$ from metals like steel is up to $1 \mathrm{MHz}$. Contribution of energy from sub-band $0-78.125 \mathrm{kHz}$ (i.e. cumulative of sub-bands 1 to 5 ) is $35 \%$ in plain concrete which is higher than reinforced concrete having $17 \%$. Contribution from sub-band $78.126-250 \mathrm{kHz}$ (i.e. cumulative of sub-bands 6 to 15 ) is $65 \%$ in plain concrete and is $83 \%$ in reinforced concrete. It is clear from Figure 7 and 8 that steel has contributed to the higher frequency bands of the signal within frequency range of used sensors. This difference in relative energy distribution can be used to detect the yielding of steel in reinforced concrete.

\section{CONCLUSIONS}

As proposed, spectral information can be utilized to give insights of fracturing material. The proposed method uses normalized energy for classification. Normalized sub-band energy is a relative measure and hence effect of attenuation and dispersion is averaged out to some extent. The method is successfully applied to differentiate AE signature of plain and reinforced concrete. It can be used to detect yielding of steel along with concrete using lower frequency range sensors. This method is useful when different $\mathrm{AE}$ sources are available in a material and classification of $\mathrm{AE}$ signals needs to be performed to know the contribution of each source in fracture. Further extensive experimental work is required to generate large set of data to adopt a library based AE signature approach for concrete.

\section{REFERENCES}

[1] Ohtsu, M., 1996. The history and development of acoustic emission in concrete engineering. Magazine of concrete research Vol. 48(177), pp. 321-330.

[2] Grosse, C.U. and Ohtsu, M., 2008. Acoustic emission testing. Springer, Heidelberg (2008).

[3] Ohno, K. and Ohtsu, M.,2010. Crack classification in concrete based on acoustic emission. Construction and Building Materials Vol. 24(12), pp. 2339-2346.

[4] Aggelis, D.G., 2011. Classification of cracking mode in concrete by acoustic emission parameters. Mechanics Research Communications Vol. 38(3), pp. 153-157.

[5] Resnikoff, H.L., Raymond Jr, O. and others, 2012. Wavelet analysis: the scalable structure of information. Springer, New York (1998).

[6] Ni, Q.-Q. and Iwamoto, M.,2002. Wavelet transform of acoustic emission signals in failure of model composites. Engineering Fracture Mechanics Vol. 69(6), pp. 717728 .

[7] Hamstad, M., O'Gallagher, A. and Gary, J., 2002. A wavelet transform applied to 
acoustic emission. Journal Acoustic Emission Vol. 20, pp. 39-61

[8] Shahiron Shahidan, Rhys Pulin, Norazura Muhamad Bunnori, Karen M. Holford., 2013. Damage classification in reinforced concrete beam by acoustic emission signal analysis. Construction and Building Materials Vol. 45, pp. 78-86

[9] Pomponi, E., Vinogradov, A. and Danyuk, A., 2015.Wavelet based approach to signal activity detection and phase picking: Application to acoustic emission. Signal Processing Vol. 115, pp. 110-119.

[10] Morizet, N., Godin, N., Tang, J., Maillet, E., Fregonese, M. and Normand, B.,2016. Classification of acoustic emission signals using wavelets and Random Forests: Application to localized corrosion, Mechanical Systems and Signal Processing Vol. 70, pp. 1026-1037.

[11] ASTM Standard, ASTM E1316-05 Standard Terminology for Nondestructive Testing. ASTM International, West Conshohocken, PA, 2005.

[12] Boyce, G. M., W. M. McCabe, and R. M. Koerner. Acoustic emission signatures of various rock types in unconfined compression. Acoustic emissions in geotechnical engineering practice. ASTM International, 1981.

[13] Mogi, Kiyoo. Study of elastic shocks caused by the fracture of heterogeneous materials and its relations to earthquake phenomena. Bull. Earthquake Res. Inst. Tokyo Univ., 40, 125, 1962.

[14] Clough, Roger B., and John A. Simmons. Reproducible acoustic emission signatures by indentation in steels. Proceedings of the DARPA/AFWAL Review of Progress in Quantitative NDE, 1981. Paper 42.

[15] N. Ativitavas. Acoustic emission signature analysis of failure mechanisms in fiberreinforced plastic structures. PhD thesis United States Texas, The University of Texas at Austin, 2002.

[16] Mirmiran, Amir, and Salam Philip. Comparison of acoustic emission activity in steel-reinforced and FRP-reinforced concrete beams. Construction and Building Materials 14.6 (2000): 299-310.

[17] Mallat, Stephane G. A theory for multiresolution signal decomposition: the wavelet representation. Pattern Analysis and Machine Intelligence, IEEE Transactions on 11.7 (1989): 674-693. 